\title{
Effect of ASE on Raman Gain in 800nm band
}

\author{
Anupam Sahu ${ }^{1}$, Sachin Kumar Gupta ${ }^{2}$, and Vijay Rao Kumbhare ${ }^{3}$
}

\begin{abstract}
In the early days of optical communication, electrical regenerators were used to amplify the optical signals. These regenerators require optical to electrical and electrical to optical conversion for amplification and transmission. To overcome the limitations of electrical regenerators, two competing technologies emerged that provide amplification in optical domain. Erbium Doped Fibre Amplifier (EDFA) and Fiber Raman Amplifier (FRA) are such two technologies that do not need conversion rather they amplify and transmit the optical signal. Initially, researchers showed great interest in EDFA. The advancement in laser technology led to the interest of FRA. Raman amplification in optical fiber has found vast amplification in optical communication because of their capability to increase transmission capacity and data rate. Fiber Raman Amplifiers has been drawing a great attention in these days. In this paper, we discussed Amplified Spontaneous Emission (ASE) generated in FRA at $808 \mathrm{~nm}$ band. In this process, a signal at $808 \mathrm{~nm}$ band is pumped at $788 \mathrm{~nm}$. The theoretical result shows that higher pump power is required to obtain the same gain while considering simulation with ASE as compared to without ASE. Furthermore, we report an additional effect of attenuation rather than amplification if there is insufficient pump power. For the conventional Single-Mode Optical Fiber (SMF) of $5 \mathrm{~km}$ length plot of gain for different pump is obtained in the MATLAB environment.
\end{abstract}

\section{Keywords- ASE, EDFA, FRA, SMF}

\section{INTRODUCTION}

$\mathrm{A}^{\mathrm{N}}$ MONG the three bands available for optical communication, $800 \mathrm{~nm}$ band has more losses. Hence, it is least preferred for communication purposes. However, it has a unique application in biomedical field. Signal in this band provide large penetrate depth into the human cells and tissue and provide optical sensing and imaging [1].

Typically, distributed Raman amplifiers have lengths greater than $40 \mathrm{~km}$ whereas discrete Raman amplifiers have lengths around $5 \mathrm{~km}$ [8]. A lumped Raman amplifier utilizes a dedicated, shorter length of fiber to provide amplification. In the case of a lumped FRA highly nonlinear fiber with a small core is utilized to increase the interaction between signals and pump wavelengths and thereby reduce the length of fiber required [5]. We discussed using distributed link for amplification

Anupam Sahu is with the Department of Electronics and Communication Engineering, MMM University of Technology, Gorakhpur-273001, (Uttar Pradesh), India (e-mail: anupam.sahu.eee11@iitbhu.ac.in).

Sachin Kumar Gupta is with the Department of Electronics and Communication Engineering, SMVD University, Katra-182320 (Jammu \& Kashmir) and Indian Institute of Technology (BHU), Varanasi-221005, (Uttar Pradesh), India (e-mail: sachin.rs.eee@iitbhu.ac.in).

Vijay Rao Kumbhare is with the Electronics and Telecommunication, J. K. Institute of Engineering, Bilaspur-495001 (Chhattisgarh), India (e-mail: vijju2486@ rediffmail.com)

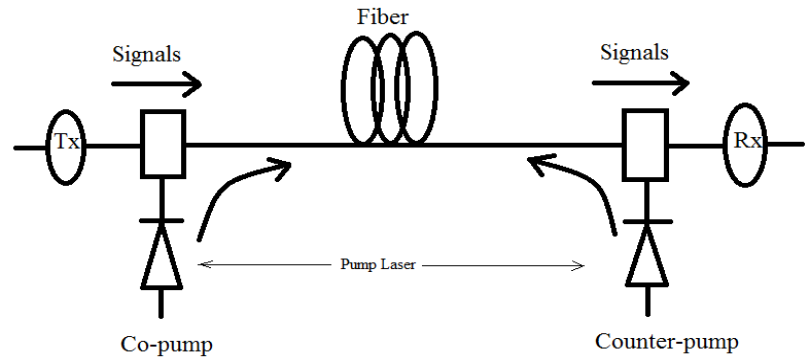

Fig. 1 Schematic showing co-pump and counter-pump

When the signal and pump enters into the medium through the same end, it is said to be forward or co-pump and when enters through opposite end, backward or counter pump as shown in fig. 1. To obtain the amplification, fiber Raman amplifier employs the intrinsic properties of silica fiber. Thus, the transmission fiber can be used as the amplification medium, where the gain is created along the transmission [8]. As the signal is tuned with respect to the pump frequency, the gain is found to be maximum at a frequency separation of $13.1 \mathrm{THz}$ between pump and signal. Two major peaks occur at $13 \mathrm{THz}$ and $15 \mathrm{THz}$ for Raman shift $\omega_{\mathrm{v}}=\omega_{\mathrm{p}}-\omega_{\mathrm{s}}$. For this shift, some miner peaks are also present in spectrum [2]. Therefore, the amorphous nature of silica is responsible for large bandwidth and multi peak nature of spectrum [8]. If the signal frequency is higher than the pump, it will suffer nonlinear absorption rather than amplification. Raman scattering is a nonlinear effect [9].

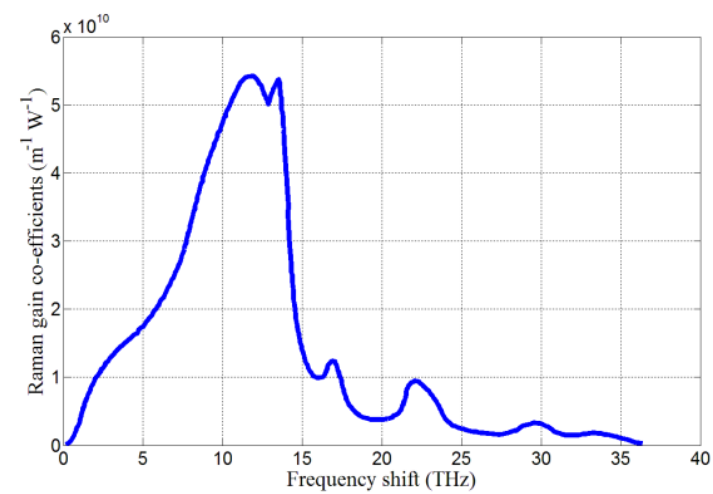

Fig. 2 Raman gain spectrum $\left(\gamma_{R}\right)$ of conventional single mode fiber (depolarized at $788 \mathrm{~nm}$ ).

In Raman amplifiers, the signal wavelength is longer than the pump wavelength by the equivalent amount of frequency shift. There are two kinds of phonons: one is acoustic phonon and the other is optical phonon. Raman scattering is the scattering between photon and optical phonon while that between photon and acoustic phonon is called Brillion scattering. Because the optical phonon has almost uniform dispersion relation versus wave number, unlike stimulated Brillouin scattering, the phase matching is 
easily obtained for arbitrary relative directions between the pump and signal waves. Therefore, Raman amplifiers can take both co- and counter pump schemes [4].

Raman amplification has advantage of self-phase matching between the pump and signal together with a broad gain- bandwidth or high speed response in comparison with the other non-linear processes [8]. One can state that the energy of a light wave is transferred to another wave, which is at a higher wavelength (lower energy) such that energy difference appears in form of phonons [3]. The other wave is known as the Stokes wave. SRS can occur in both the direction whereas SBS only in forward direction.

\section{Mathematical Modelling And Prediction}

When stronger pump is applied along with the weaker signal, the signal will be amplified due to SRS. The signal amplification is described by the following equations:

$$
\begin{aligned}
& \frac{d P_{s}}{d z}=-\alpha_{s} P_{s}+\left(C_{R}\right) P_{p} P_{s} \\
& \xi \frac{d P_{P}}{d z}=-\alpha_{p} P_{P}-\frac{\omega_{p}}{\omega_{s}}\left(C_{R}\right) P_{p} P_{S}
\end{aligned}
$$

Where, the product term containing pump power and signal power describes the coupling effect via SRS. This coupling effect is determined by the efficiency of Raman gain in the fiber $C_{R}$, which is defined as normalized gain with the effective mode area of effective mode are of 19.625 $\mu \mathrm{m}^{2}$ for SMF. Raman gain Coefficient of $5.4 \times$ $10^{-17} \mathrm{~km} / W$ is used for observation, $\alpha_{s}$ and $\alpha_{p}$ are the attenuation coefficient at the pump and signal wavelength, $P_{p}$ and $P_{s}$ are the pump and signal power, $\omega_{p}$ and $\omega_{s}$ are angular frequencies of pump and signal. The $\xi= \pm$ represent the forward and backward pump respectively.

The first term of the equation (1) and (2) represents the intrinsic signal (pump) loss and second term represents the signal gain (pump depletion) due to SRS. Consider the forward pump propagation; the second term of equation (1) can be neglected by setting $g_{R}=0$ for small signal amplification:

$$
\begin{aligned}
& P_{p}=P_{p}(0) e^{-\alpha_{p} z} \\
& P_{S}=P_{S}(0) \exp \left[\left(C_{R}\right) \frac{P_{p}(0)}{\alpha_{p}}\left(1-e^{-\alpha_{p} z}\right)-\propto_{s} z\right]
\end{aligned}
$$

$P_{0}=P_{p}(0)$ is the input pump power and $L_{e f f}$ is the effective length and it is given by

$$
L_{e f f}=\left[1-e^{-\propto_{p^{z}}}\right] / \propto_{p}
$$

\section{A. Amplifier Gain}

Amplifier gain can be defined as the ratio of output power with amplification to the output power without amplification.

$$
G_{A}=\frac{P_{S}(L)}{P_{S}(0) \exp \left(-\alpha_{s} L\right)}=\exp \left(g_{0} L\right)
$$

Where the small-signal gain $g_{0}$ is defined as

$$
g_{0}=C_{R} P_{o}\left(\frac{L_{e f f}}{L}\right) \approx \frac{C_{R} P_{0}}{\alpha_{p} L}
$$

Raman gain is polarization dependent [6]. The coupling effect between signal and pump is stronger if they are co- polarized than if they are orthogonally polarized. The polarization factor is $K$, which is 1 if the pump and signal waves are polarization matched, or 2 if depolarized.

$$
G_{A}=4.34\left(C_{R} L_{e f f} \frac{P_{0}}{K}-\propto_{s} L\right)
$$

\section{B. Multiple Signal Propagation Couple Equation:}

Solving the propagation equation for pump, single signal and forward ASE as boundary value problem. Wave propagation equation for fiber Raman amplifier with multiple signal and pump channels can be described by the following system of coupled nonlinear equations:

$$
\pm \frac{d P_{k}}{d z}=-\propto_{k} P_{k}+\rho P_{k}+\sum_{j=1}^{n_{p}+n_{s}} \gamma\left(v_{j}, v_{k}\right) P_{k} P_{j}
$$

Where $n_{p}$ is the number of pump waves and $n_{s}$ is the number of signal channels, subscripts $j, k$ denote the $j$ th $\left(j=1,2, \ldots, n_{p}+n_{s}\right)$ and $k$ th $\left(k=1,2, \ldots, n_{s}\right)$ waves. $P_{k}, P_{A S E, k}$ represents the $k$ th signal and amplified spontaneous emission (ASE) power, while $P_{j}$ can the signal or pump power. $z$ is the distance and the \pm sign denotes the propagation directions.

Neglecting the Rayleigh back scattering of ASE noise, analysis of ASE noise is based on a set of couple steadystate equations [12]. The forward and backward power evolution of ASE noise can be expressed in terms of the following equations.

$$
\begin{aligned}
& \pm \frac{d P_{A S E, k}}{d z}=-\propto_{k} P_{A S E, k}+\rho P_{A S E, k}+\sum_{j=1}^{n_{p}+n_{s}} \gamma\left(v_{j}, v_{k}\right) P_{j} \\
& \times\left[P_{A S E, k}+2 h v_{k} \Delta v\left(1+H_{j k}\right)\right]
\end{aligned}
$$

Where $P_{A S E, k}$ is the ASE noise optical power variable $v, \propto, \rho, h$ and $\Delta v$ are the optical frequency, fiber attenuation, Rayleigh scattering coefficient, Plank's constant, and effective ASE bandwidth, respectively.

In the right of the equations, the first term represents the attenuation of the fiber, the second term represents Rayleigh scattering of ASE optical waves, the third term represents the stimulated Raman scattering (SRS) between pump (signal) waves and ASE waves, the fourth term represents the spontaneous Raman scattering of pump (signal) waves and it is the source of noise. The boundary condition of the equation are $\mathrm{P}_{\mathrm{ASE}, \mathrm{k}}(0)=0$ and $\mathrm{P}_{\mathrm{ASE}, \mathrm{k}}(\mathrm{L})=\mathrm{L}$. The conventional SMF with effective mode area of $12.56 \times 10^{-18} \mathrm{~km}^{2}$ with single signal and pump is observed in matlab environment. Because more power of pump is required, hence more amplified spontaneous emission (ASE) is generated in the given amplifier. In addition, the input signal power to the amplifier has lowered down. This lower signal power means that the ASE can more successfully compete with the signal for gain in the amplifiers [7].

\section{Simulation Results AND Discussion}

The simulation is performed under MATLAB environment. For a conventional SMF fiber length of $5 \mathrm{~km}$, attenuation coefficients of $0.78 \mathrm{~dB} / \mathrm{km}$ for both pump \& signal and are taken into account. 


\section{A. For propagation Coupled equation without ASE}

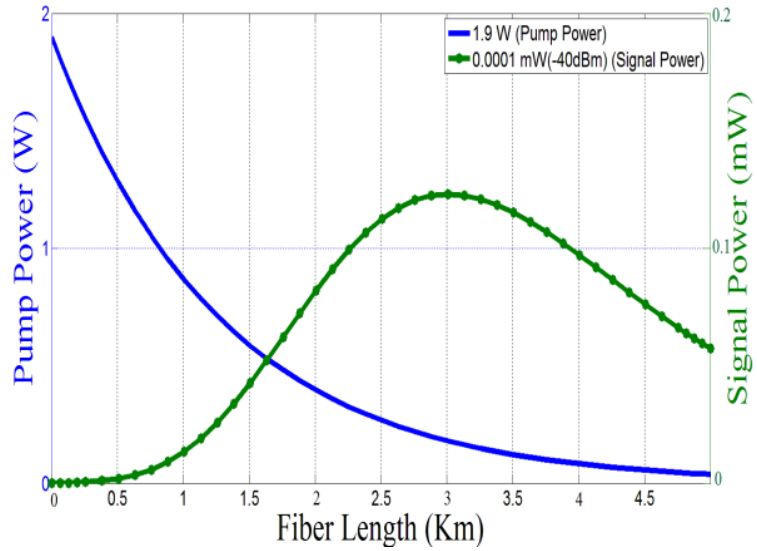

Fig. 3 Variation of optical power with the fiber length for a fiber Raman amplifier

The co-pumped signal is amplified along the medium with a peak at about $\mathrm{L}=3 \mathrm{~km}$ and then it suffers attenuation due to insufficient pump power. The pump continuously delivers its power to the signal and after certain distance; it falls below the certain threshold limit. The signal then suffers attenuation with its attenuation coefficient. Minimum threshold pump required to attain amplification is $0.2 \mathrm{~W}$. Maximum gain of approximately $30.90 \mathrm{~dB}$ is observed at a link length of $\mathrm{L}=3 \mathrm{~km}$.

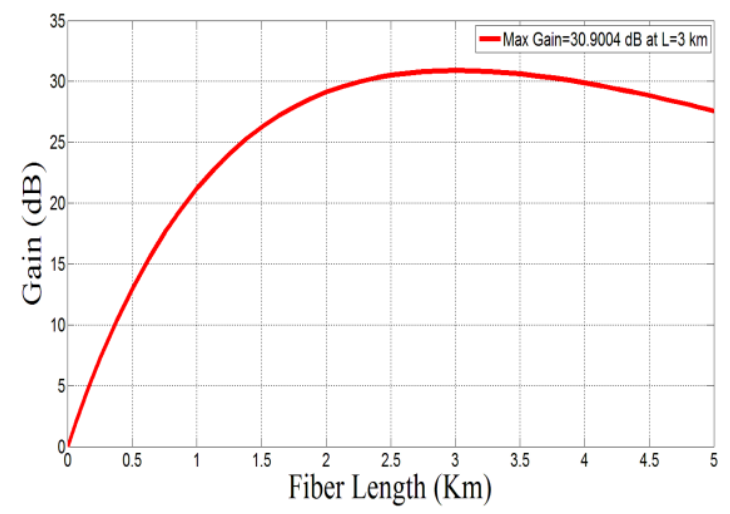

Fig. 4 Variation of signal gain with the fiber length

As shown in Fig. 5, as the pump power increases corresponding gain spectrum also increases. For pump power less than $0.2 \mathrm{~W}$, the above figure shows the attenuation. Hence, a minimum pump of $0.2 \mathrm{~W}$ is required for amplification.

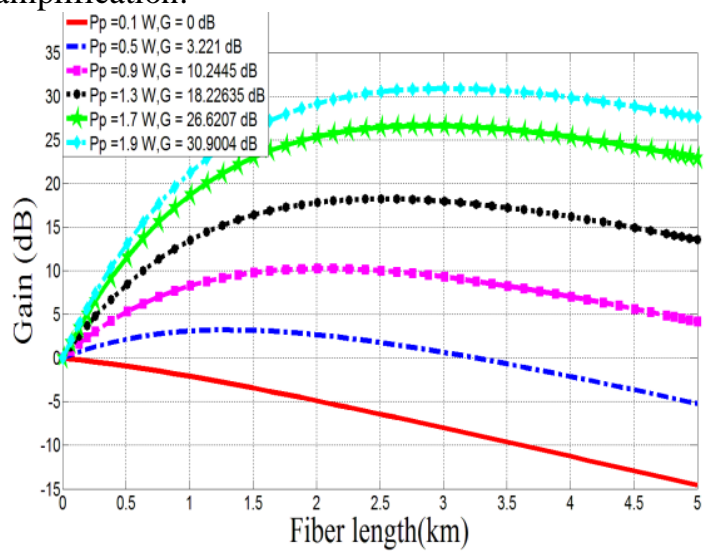

Fig. 5 Variation of gain with fiber length for different pump power

\section{B. Simulation result for propagation coupled equation with} ASE:

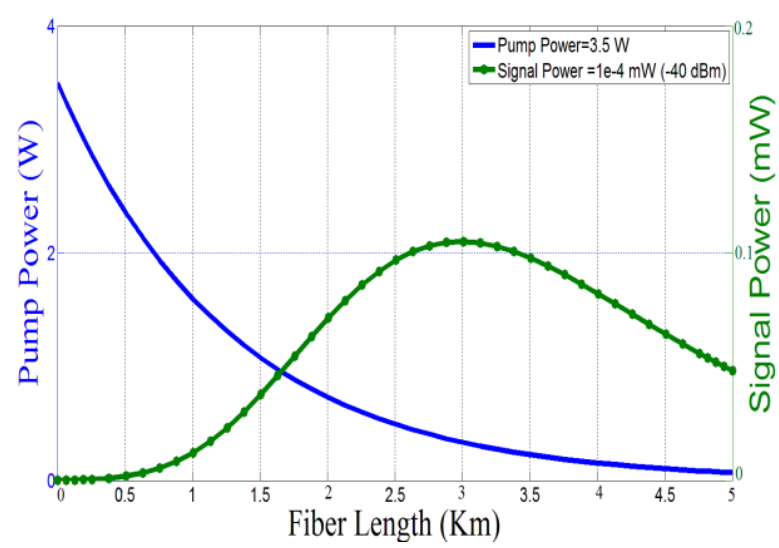

Fig. 6 Variation of optical power with the fiber length with ASE

The ASE with signal strength of $-70 \mathrm{dBm}$ and bandwidth of $0.125 \mathrm{THz}$ degrades the signal strength along the length and hence, higher thresholds pump of $0.4 \mathrm{~W}$ is required

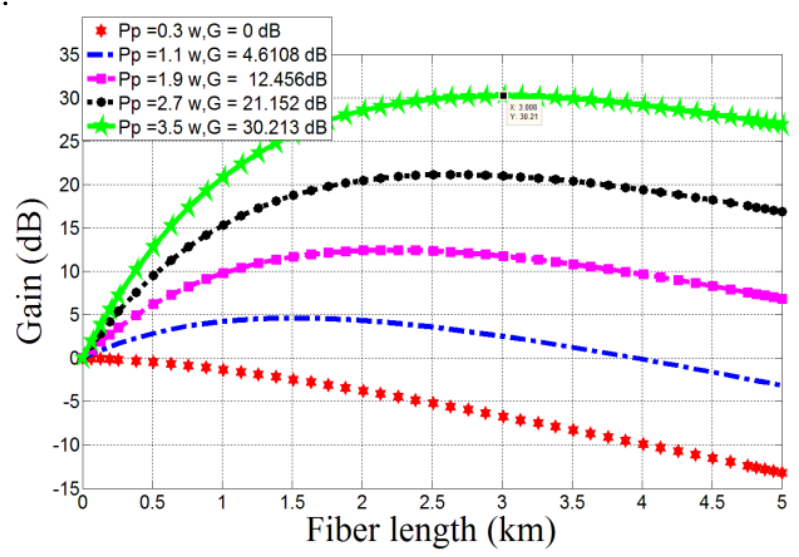

Fig. 7 Variation of signal gain with the fiber length for different input pump power

As shown above, as the pump increases, gain spectrum also increases but to obtain the same gain of approximately $30 \mathrm{~dB}$, pump of $3.5 \mathrm{~W}$ is required, which is much greater than previous. For pump power less than $0.4 \mathrm{~W}$, signal attenuates rather than amplification.

\section{CONCLUSION}

By solving the propagation-coupled equation in MATLAB environment for single pump at $788 \mathrm{~nm}$ and single signal at $808 \mathrm{~nm}$ without ASE, it is found that the threshold power for the single pump and single signal is $0.2 \mathrm{~W}$. When the pump power is greater than threshold, it amplifies the signal. During amplification, pump continuously loses its power though its attenuation coefficient. As it passes the threshold it no longer amplifies the signal, rather absorbs. On varying the pump from $0.1 \mathrm{~W}$ to $1.9 \mathrm{~W}$, we achieved a maximum gain of $30.90 \mathrm{~dB}$ at fiber length of $3 \mathrm{~km}$ with pump power of $1.9 \mathrm{~W}$. Similarly, for the single pump and single signal with ASE amplifier system threshold power obtained is $0.4 \mathrm{~W}$. This higher value of threshold is due to presence of ASE noise, whose effect increases with the increase in pump power. On varying the pump from $0.3 \mathrm{~W}$ and above, we achieved the same gain of approximately $30.213 \mathrm{~dB}$ at a fiber length of $3 \mathrm{~km}$ with pump 
power of $3.5 \mathrm{~W}$. Therefore an additional pump of $1.6 \mathrm{~W}$ is required to achieve the same gain at the same point of length. This additional requirement of pump power is due the presence of ASE.

\section{REFERENCES}

[1]. A. Mahjoubfar, K. Goda, and B. Jalali, "Raman amplification at 800 $\mathrm{nm}$ in single-mode fiber for biological sensing and imaging", in Conference on Lasers and Electro-Optics, OSA Technical Digest (CD) (Optical Society of America, 2010). https://doi.org/10.1364/cleo.2010.cfa4

[2]. R. W. Boyd, "Nonlinear Optics", Academic Press, SanDiego, CA, 1992.

[3]. K. Okamoto, "Theory of Optical Waveguides. Tokyo," Corona, Japan pp. 191-198, 1992.

[4]. M. N. Islam, "Raman Amplifiers for Telecommunications 1: Physical Principles", 2003.

[5]. J. Bromage, "Raman Amplification for Fiber Communication Systems", IEEE Journal of Lightwave Technology, vol. 22, no. 1, pp. 79-93, January 2004. https://doi.org/10.1109/JLT.2003.822828

[6]. S. P. Singh, R. Gangwar, and N. Singh, "Nonlinear Scattering effect in optical Fibers", progress in electromagnetic research, PIER 74, pp. 379-405, 2007. https://doi.org/10.2528/PIER07051102

[7]. C. Headley, G. P. Agrawal, "Raman Amplification in fiber optical communication system", 2005.

[8]. G. P. Agrawal, "Fiber-Optic Communication Systems," 2 , Wiley, New York, NY, 1997.

[9]. C. Kakkar, K. Thyagarajan, "Novel fiber design for flat Gain Raman amplification using single pump and dispersion compensation in $\mathrm{S}$ band", Journal of light wave technology, vol. 22, No. 10, October 2004. 